\title{
The Patient-Doctor Relationship and Online Social Networks: Results of a National Survey
}

Dominick L. Frosch, PhD

Palo Alto Medical Foundation Research Institute, Palo Alto, CA, USA.

J Gen Intern Med 27(4):404

DOI: $10.1007 / \mathrm{s} 11606-012-1990-\mathrm{Z}$

(c) Society of General Internal Medicine 2012

The Editors Reply-We appreciate the thoughtful and valid critique of this study by Dr. Campbell and colleagues.

The low response rate of the survey was indeed the primary critique of this study during peer review. However, this critique was balanced by the novelty of the topic and relative lack of published data. Reviewers, therefore, suggested focusing the discussion of results on survey questions related to the ethics of online social network use

(OSN). These questions are presumed to be less susceptible to bias than questions related to prevalence of OSN use.

The published manuscript reflects these suggestions and we believe is appropriately cautious about the clear and important limitations of this study. More rigorous surveys are clearly needed to quantify physician OSN use at the national level. But the paper by Bosslet and colleagues suggests interesting domains to include in future investigations.

Corresponding Author: Dominick L. Frosch, PhD; Palo Alto Medical Foundation Research Institute, Palo Alto, CA, USA (e-mail: froschd@pamfri.org). 